\title{
Disordered and Densely Packed ITO Nanorods as an Excellent Lithography-Free Optical Solar Reflector Metasurface
}

\author{
Deniz Umut Yildirim, ${ }^{*},+, \oplus$ Amir Ghobadi, ${ }^{\dagger,}$ Mahmut Can Soydan, ${ }^{\dagger, \dagger}$ Okan Atesal, ${ }^{\dagger}$ Ahmet Toprak, ${ }^{\dagger}$ \\ Mehmet Deniz Caliskan, ${ }^{\dagger}$ and Ekmel Ozbay ${ }^{*, \dagger, \$, \S, \|}$
}

${ }^{\dagger}$ NANOTAM-Nanotechnology Research Center, ${ }^{\star}$ Department of Electrical and Electronics Engineering, ${ }^{\S}$ Department of Physics, and "UNAM-Institute of Materials Science and Nanotechnology, Bilkent University, 06800, Ankara, Turkey

Supporting Information

ABSTRACT: Precise control and stabilization of the operating temperature environment of spacecraft and satellites during their life cycle is of paramount importance to increase device reliabilities and reduce the thermomechanical constraints. Optical solar reflectors are the physical interface between the spacecraft and space, and they are broadband mirrors for the solar spectrum, while having strong thermal emission in the mid-infrared part of the electromagnetic spectrum. Strong light-matter interactions in metamaterials and metasurfaces offer significant advantages compared to the conventional methods in performance, weight, launch, and assembly costs. However, the fabrication complexity of these metastructures due to necessitating lithography hinders their upscaling, reproducibility, large-area compatibility, and mass production. In this regard, we propose a facile,

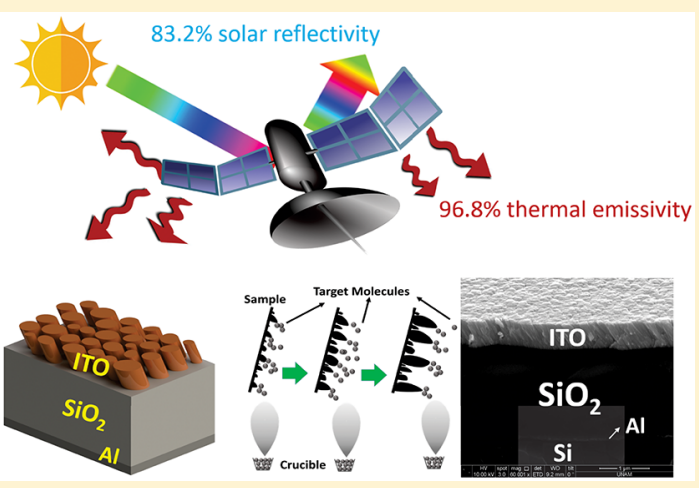
lithography-free fabrication route, exploiting oblique deposition to design a metasurface based on disordered and densely packed Indium Tin Oxide (ITO) nanorod forests. The excellent light trapping capability of the nanorod forests, randomness in the geometrical dimensions of these nanorods, combined with the lossy plasmonic nature of ITO in the thermal-infrared range led to strong coupling of thermal radiation to broad plasmonic resonances and, consequently, an experimental emissivity of 0.968 , in a very wide range from 2.5 to $25 \mu \mathrm{m}$. In the solar spectrum, the low-loss dielectric characteristic of ITO resulted in an experimental solar absorptivity as small as 0.168 . Our proposed design with high throughput, robustness, low cost, and high performance, therefore, shows great promise not only for space missions, but also for promoting environmentally friendly passive radiative cooling for our planet and thermal imaging in the field of security labeling.

KEYWORDS: metasurfaces, oblique-angle deposition, metamaterial perfect absorbers, optical solar reflectors, transparent conductive oxides, plasmonics

$\mathrm{T}$ emperature stabilization and its uniform distribution are vital for spacecraft and satellites because most of their components become less reliable when operated outside of their acceptable temperature range. Propellant freezing, thermal cycling damage to the equipment, and instrument/ antenna/camera misalignment are additional issues to be avoided during all of the mission phases. ${ }^{1-3}$ The coating system called Optical Solar Reflectors (OSRs), which are secondary-surface mirrors, therefore, play a crucial role for the optimum performance of spacecraft and satellites and success of their missions. ${ }^{1-6}$ OSRs simultaneously minimize the direct and indirect solar energy input to the spacecraft while passively emitting the thermal energy generated on the board. OSRs are then required to have a small solar absorptance $\left(\alpha_{\mathrm{s}}\right)$ over the blackbody radiation spectrum of sun at $5778 \mathrm{~K}$, corresponding to the ultraviolet (UV), visible (VIS), and near-infrared (NIR) parts of the electromagnetic spectrum. Concurrently, OSRs should have a large thermal emissivity $\left(\epsilon_{\mathrm{IR}}\right)$ in the mid-infrared (MIR) and far-infrared (FIR) parts of the spectrum (thermal- infrared) related to the blackbody radiation at room temperature, $300 \mathrm{~K}$. OSRs can be regarded as spectrally selective filters, that is, they are broadband mirrors in the solar spectrum, but perfect absorbers in the thermal infrared. A Figure of Merit (FoM) for an OSR can, therefore, be defined as $\epsilon_{\mathrm{IR}} / \alpha_{\mathrm{s}}$. Another critical consideration for an OSR is the stability over the course of the mission. This is because an OSR is affected during their missions by contamination, highvacuum, UV radiation, and charged particles. The general result is an increase in $\alpha_{s}$, with a negligible effect on $\epsilon_{\mathrm{IR}}$. There are different strategies and material choices to design an OSR, and they can be classified under two categories: conventional methods of using white paint, second-surface mirrors using quartz or Teflon; ${ }^{1-3}$ and metamaterial-based solutions. ${ }^{5,6}$

Under the category of conventional methods, white color is often painted on the exterior surfaces and radiators of a

Received: April 29, 2019

Published: June 18, 2019 

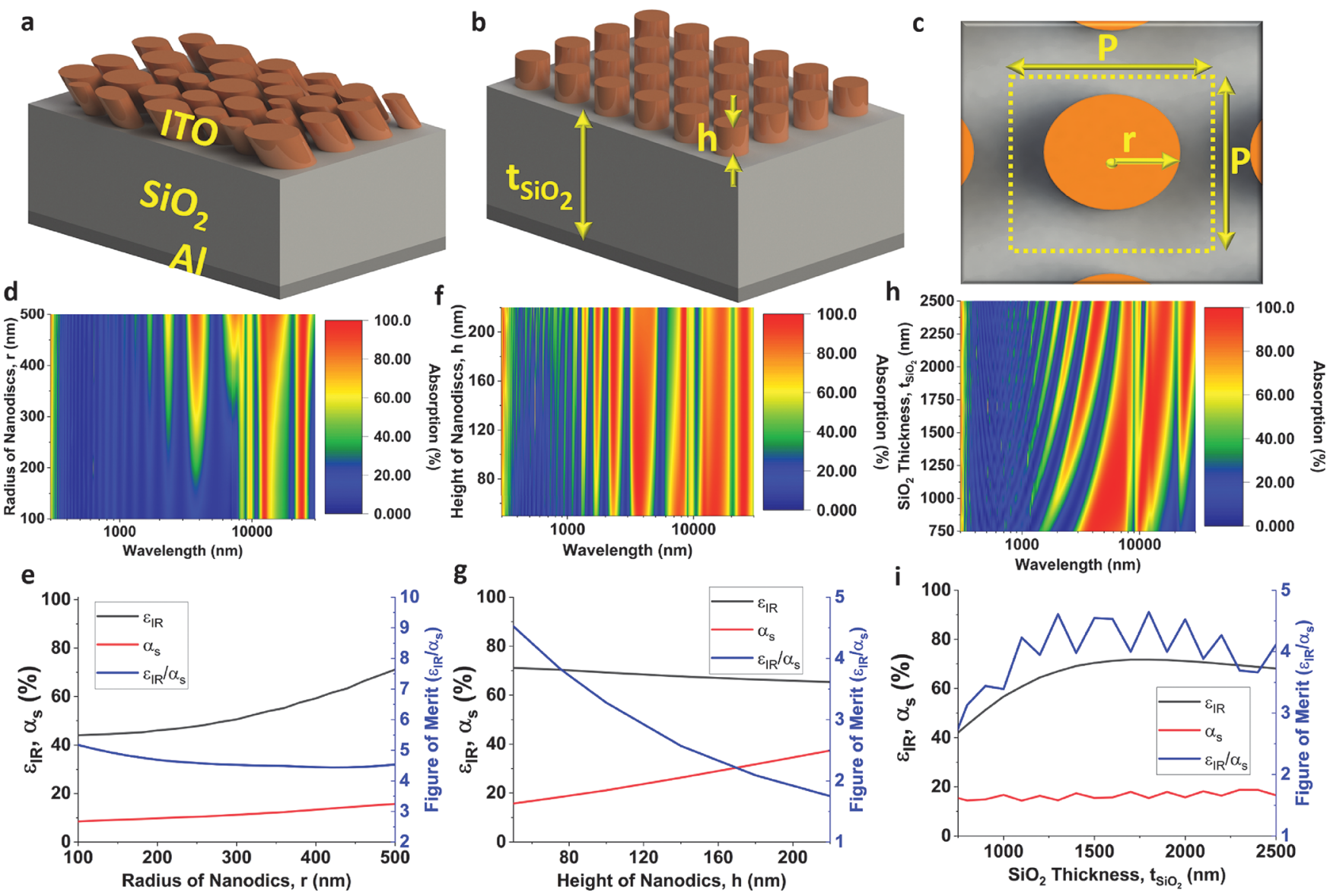

Figure 1. Schematics of Devices 1 and 2 and the parameter sweeps on the geometrical dimensions of Device 2. (a) Device 1 to achieve broadband absorption in thermal-infrared and broadband reflection in solar spectrum. (b) Device 2 to test the effect of geometrical dimensions of a nanodisc on absorption spectrum, thermal emissivity, and solar absorptivity. (c) Top-view of Device 2, showing the geometrical parameters in detail. Absorption spectrum of Device 2 under varying (d) nanodisc radius, $r$, (f) thickness of nanodiscs, $h$, and (h) thickness of the $\mathrm{SiO}_{2}$ spacer, $t_{\mathrm{SiO}_{2}}$. OSR parameters Device 2 when (e) $r,(\mathrm{~g}) h$, and (i) $t_{\mathrm{SiO}_{2}}$ are changed.

spacecraft due to having a large $\epsilon_{\mathrm{IR}} / \alpha_{\mathrm{s}}$ ratio. However, these paintings are very prone to environmental degradation due to UV radiation that darkens the pigments. ${ }^{1-3}$ Second-surface reflectors based on silver (Ag)- or aluminum ( $\mathrm{Al}$ )-backed quartz films combine the high reflection of the metal layer with the strong absorption/emission of quartz in the thermal infrared to achieve very high $\epsilon_{\mathrm{IR}} / \alpha_{\mathrm{s}}$. In addition, quartz is highly insensitive to UV radiation and darkening, so it has very stable thermo-optical properties. Nevertheless, having large emissivity in the thermal infrared range requires using thick tiles of quartz ranging from $250 \mu \mathrm{m}$ to a few $\mathrm{cm}$. Moreover, the rigid and brittle tiles are prone to breaking while handling and are inflexible. This adds significant weight and, consequently, launch and assembly costs for the spacecraft. The other type of secondary-surface mirrors replace quartz with fluorinated ethylene propylene (FEP) Teflon film. The resultant Ag/FEP and $\mathrm{Al} / \mathrm{FEP}$ materials provide flexibility and an excellent beginning-of-life $\epsilon_{\mathrm{IR}} / \alpha_{\mathrm{s}}$ ratio. However, FEP is highly susceptible to degradation during its lifespan, primarily due to charged-particle damage and atomic oxygen. This makes the lifespan of $\mathrm{Ag} / \mathrm{FEP}$ and $\mathrm{Al} / \mathrm{FEP}$ materials shorter compared to quartz tiles. ${ }^{1-5}$ Thus, the conventional methods cannot simultaneously offer high performance, low weight, and the associated costs at the same time.

The advances in nanofabrication in the last few decades provided the opportunity to observe strong light-matter interactions in synthetic, man-made materials known as metamaterials whose subwavelength inclusions offer exotic properties, such as negative refraction, ${ }^{7,8}$ artificial magnetism, ${ }^{9,10}$ asymmetric transmission, ${ }^{11,12}$ cloaking, ${ }^{13,14}$ and lasing. ${ }^{15,16}$ Their subwavelength thick counterparts, which are called metasurfaces, are also finding increased applications in ultrathin flat lenses, spatial light modulators, spectral filters, and polarization control. ${ }^{17-24}$ Strong light-matter interactions also enable the harvesting of the confined electromagnetic radiation by an absorbing layer such as metals, or semiconductors, which could lead to the realization of perfect absorption of light. ${ }^{25,26}$ This resonant response can be narrowband, which has applications in sensing, imaging, and color filtering. ${ }^{27-35}$ On the other hand, their broadband counterparts are promising in the areas of thermal photovoltaics, ${ }^{36,37}$ hot-electron-based photodetectors, ${ }^{38,39}$ and, in most relations to OSRs, radiative cooling. ${ }^{5,6,40-45}$

Attempts on achieving broadband absorption mostly focused on metal-insulator-metal (MIM)-based cavities, with topmetal layer patterns of trapezoids, ${ }^{46,47}$ nanodiscs, ${ }^{48,49}$ pyramids, ${ }^{50}$ gratings, ${ }^{51}$ nanopatches, ${ }^{52-54}$ multiple narrowband resonators, ${ }^{55}$ elongated shapes, ${ }^{56}$ and tapered structures. ${ }^{57-61}$ Nonetheless, the broadband absorption comes at the expense of requiring a lithography step during fabrication. This constitutes a major complication when applications for spacecraft coating are considered, due to not being large-area 
compatible, and not offering mass-production with highthroughput, upscaling, and repeatability. One solution to this problem can be utilizing planar multilayer designs. ${ }^{62-65}$ However, these designs only rely on interference and having a broad absorption in thermal infrared may require multiple deposition cycles with large thickness, resulting in bulky devices. As an alternative method, disorder and randomness are shown to result in a broader absorption spectrum compared to planar designs, which is due to the light trapping and confinement in nano units, as well as exciting multiple resonances whose superposition contribute to a broad response. ${ }^{66-70}$ Nevertheless, these designs require multiple fabrication steps and utilize slow chemical processes, so there is still room for improvement in achieving disorder and randomness in the devices with higher throughput. OSR applications also place major restrictions on the material choices because the requirement of simultaneously realizing solar reflection and a broadband absorption in thermal infrared.

Recently, transparent conductive oxides (TCOs), such as indium tin oxide (ITO), Aluminum-doped $\mathrm{ZnO}$ (AZO) and gallium-doped $\mathrm{ZnO}$ (GZO) emerged as strong candidates for infrared plasmonics, while being highly transparent in the solar spectrum. ${ }^{71-77}$ Their relatively high loss in thermal infrared promises plasmonic resonances with low quality factors and a broad absorption spectrum. Conversely, their low-loss dielectric response in the visible range would not contribute significantly to absorption. This means high $\epsilon_{\mathrm{IR}}$, but low $\alpha_{\mathrm{s}}$ are simultaneously achievable. Indeed, an AZO-based OSR with a square-shaped unit cell is recently demonstrated ${ }^{5}$ with an $\epsilon_{\mathrm{IR}} /$ $\alpha_{\mathrm{s}}$ ratio as high as 4.9 , although electron-beam lithography (EBL) is employed in the fabrication. As a result, a lithography-free route is still sought for large-scale compatibility.

In the present paper, we use disordered and densely packed Indium Tin Oxide (ITO) nanorod forests as the top layer of a metal-insulator-oxide cavity. The outstanding light trapping capability of the scaffold increased the residing time of thermal radiation in the device. This trapped light is then harvested by the hybrid system of ITO and $\mathrm{SiO}_{2}$. As a result, perfect broadband absorption over the entire thermal-infrared, ranging from 2.5 to $25 \mu \mathrm{m}$ is achieved and the experimental $\epsilon_{\mathrm{IR}}$ reached as high as 0.968 . At the same time, solar absorptance, $\alpha_{\mathrm{s}}$, is also minimized to an experimental value of 0.168 , owing to the lowloss dielectric characteristic of ITO in this spectral range. In fabricating the nanorod forests, we exploited the inherent lineof-sight type coating of Physical Vapor Deposition (PVD) systems, such as thermal evaporator and sputtering, ${ }^{78}$ to eliminate the need for lithography. In the method, which is known as oblique-angle deposition, ${ }^{79-82}$ the substrate is placed at an angle to the source, enabling the creation of quasi-3D structures ${ }^{83,84}$ with drastically different properties. ${ }^{85-87}$ The paper is organized as follows: In the first part of this paper, we utilize the finite-difference-time-domain (FDTD) method to numerically investigate broadband light absorption in thermalinfrared and broadband reflection in solar spectrum, in a metasurface comprised of periodic nanodiscs. We scrutinize the effects of the geometrical dimensions of a single nanodisc, and cavity thicknesses on absorption spectrum, $\epsilon_{\mathrm{IR}}, \alpha_{\mathrm{s}}$, and FoM, to shed light on optimizing the actual device. In the second part, we demonstrate our oblique-angle deposition strategy in detail and present the experimental results for the optimized OSR. We compare these results with the numerical results of the periodic disc design and experimental results for designs utilizing planar ITO. Overall, our robust, large-area compatible and ultrahigh performance OSR holds great promise in not only space missions, but also in radiative cooling and thermal imaging.

\section{RESULTS AND DISCUSSION}

Theoretical and Numerical Design and Optimization of the OSR Metasurface. Our proposed device in Figure 1a is designed to obtain the required spectral response of an OSR, that is, broadband reflection in the solar spectrum, while achieving broadband absorption in the thermal-infrared, due to its highly diffracting/scattering and electromagnetic wave (EMW) trapping nature. This proposed structure, which we call Device 1 , consists of a metal-insulator-oxide (MIO) cavity, where a thick metal back-reflector, aluminum ( $\mathrm{Al})$, is used to redirect the otherwise transmitted EMWs back into the cavity. The spacer, $\mathrm{SiO}_{2}$, is used to create the necessary phase accumulation to redirect the EMWs mostly to the top absorbing layer of our MIO cavity, which makes use of ITO nanorods. We also outlined the reasons of picking these materials in more detail in the Supporting Information. ITO is a plasmonic material in the thermal-infrared, so the nanorods can be used to excite localized surface plasmon resonances (LSPRs). LSPRs define the nonpropagating excitations of the conduction electrons that are coupled to the incident electromagnetic radiation. When the nanoparticle dimensions become comparable to wavelength, the plasmons are confined to the surface of nanoparticles and the collective harmonic oscillations result in a dipolar response with a specific resonance wavelength. The resonance wavelength and its $Q-$ factor are highly related to the morphology (size, shape, spacing, and density) of the nanostructures. ${ }^{88}$ Therefore, in understanding the operation of Device 1, it is pivotal to first grasp how individual nanorod dimensions and the cavity thickness affect the spectral response as well as the OSR parameters, $\epsilon_{\mathrm{IR}}, \alpha_{\mathrm{s}}$ and $\epsilon_{\mathrm{IR}} / \alpha_{\mathrm{s}}$.

To understand how the performance is affected by the geometrical parameters, we initially scrutinized the LSPRs supported by nanodiscs/nanorods of radius $r$, thickness $h$, that are periodic in two directions with a period of $P$, when the spacer thickness is chosen as $t_{\mathrm{SiO}_{2}}$. We name this testing device as Device 2, whose schematic is shown in Figure 1b, and its unit-cell from the top view is shown in Figure 1c. To clarify the difference between Devices 1 and 2, Device 1 is the device that will be fabricated and characterized in the upcoming parts of this paper. It has nanorods whose size, shape, radii, and spacing are defined randomly during the fabrication, while $h$ is controlled by deposition rate and time. On the other hand, Device 2 has periodic nanodiscs with precise radii and spacing, which can be controlled by EBL. It is defined to compare the optical performance of a patterned top layer to that of Device 1.

In the numerical analysis of Device 2, we utilize finitedifference-time-domain (FDTD) simulations. Details of the simulation setup are outlined in the Experimental Section. With the aid of FDTD method, we calculated the spectral absorption, $A(\lambda, T)$, of Device 2. Based on the calculated absorption data and the spectral radiance $B(\lambda, T)$, given in eq 1 , the $\epsilon_{\mathrm{IR}}$ and $\alpha_{\mathrm{s}}$ values are calculated based on eq 2 . 


$$
\begin{aligned}
& B(\lambda, T)=\frac{2 h c^{2}}{\lambda^{5}} \frac{1}{e^{h c / \lambda k_{\mathrm{b}} T}-1} \\
& \epsilon_{\mathrm{IR}}, \alpha_{\mathrm{s}}=\frac{\int_{\lambda_{1}}^{\lambda_{2}} A(\lambda, T) B(\lambda, T) \mathrm{d} \lambda}{\int_{\lambda_{1}}^{\lambda_{2}} B(\lambda, T) \mathrm{d} \lambda}
\end{aligned}
$$

where $h, c$, and $k_{\mathrm{b}}$ are Planck's constant, speed of light in vacuum, and Boltzmann constant, respectively. The integration limits $\lambda_{1}$ and $\lambda_{2}$ are taken as $300-2500 \mathrm{~nm}$ and $2.5-25 \mu \mathrm{m}$, respectively, for the solar spectrum and thermal infrared. $\lambda$ is the spectral wavelength and $\mathrm{T}$ is the temperature, which is $5778 \mathrm{~K}$ for solar spectrum and $300 \mathrm{~K}$ for the thermal radiation. Equation 2 then represents the spectrally weighted absorbance of the metasurface OSR. In the numerical simulations, the dispersion effect of $\mathrm{Al}, \mathrm{SiO}_{2}$, and ITO are retrieved with ellipsometry measurements, and they are plotted in Figure S4.

We first fixed $t_{\mathrm{Al}}$ at $125 \mathrm{~nm}$ (throughout this paper), $P$ at $1100 \mathrm{~nm}, h$ at $50 \mathrm{~nm}, t_{\mathrm{SiO}_{2}}$ at $2000 \mathrm{~nm}$, and swept $r$. Our primary purpose in these set of simulations is to find out the effect of the density or fill factor of the nanodics in the absorption spectrum and the OSR parameters. In Figure 1d we show the spectral response of Device 2 for different values of $r$. First, it is seen that the high-loss plasmonic optical nature of ITO in thermal infrared results in a broad absorption spectrum, while in the solar spectrum, the oscillations in absorption corresponds to response of a Fabry-Pérot cavity, due to ITO's low-loss dielectric nature. Figure le shows how OSR parameters respond to changes in $r$. In the thermalinfrared, it is evident that, as the nanodiscs get closer to each other with decreasing $r$, in other words, as the discs become denser, their "interconnectedness" increases and the coupling of dipole oscillations become stronger, ${ }^{89}$ and broad LSPRs can be supported. In the solar spectrum, an increase in $r$ corresponds to a larger fill factor of ITO compared to vacuum, so the dielectric losses increase. The increase in the $\alpha_{\mathrm{s}}$, nevertheless, outweighs the increase in $\epsilon_{\mathrm{IR}}$, so the FoM, $\epsilon_{\mathrm{IR}} / \alpha_{\mathrm{s}}$ is essentially larger for the more isolated nanodiscs. We also varied the periodicity, $P$, of the unit cell with a fixed $h$ of 50 $\mathrm{nm}, t_{\mathrm{SiO}_{2}}$ of $2000 \mathrm{~nm}$ and $r$ of $200 \mathrm{~nm}$. Our treatment based on the density of the nanodiscs also held true for the variable periodicity, and the results are demonstrated in Figure S5a,b.

Next, we investigated the role of height of each nanodisc in the absorption spectrum and the OSR parameters. For this purpose, we kept $P$ at $1100 \mathrm{~nm}, r$ at $500 \mathrm{~nm}, t_{\mathrm{SiO}_{2}}$ at $2000 \mathrm{~nm}$, and swept $h$. We preferred to keep the nanodiscs densely packed as it imitates our proposed densely packed nanorods better. The absorption spectrum for different values of Figure If shows that, in the solar spectrum, the height causes an increase in the absorption because the EMWs interact more with ITO before reaching the cavity. In thermal-infrared though, longer nanodiscs caused a decrease in the absorption strength, as the strength of EMWs reaching to the cavity is now reduced, and reflection due to ITO is increased. ${ }^{56}$ The OSR parameters based on our findings is plotted in Figure 1g, which clearly shows that a thin layer of ITO is beneficial for both $\epsilon_{\mathrm{IR}}$ and $\alpha_{\mathrm{s}}$, consequently, for the figure of merit as well.

The cavity thickness, $t_{\mathrm{SiO}_{2}}$ is of fundamental importance in an MIM cavity configuration. For our design, we used a Salisbury screen configuration, ${ }^{90}$ with a thickness of $\lambda_{0} / 4 n_{\mathrm{SiO}_{2}}$, where $n_{\mathrm{SiO}_{2}}$ is the real part of the complex refractive index of
$\mathrm{SiO}_{2}$. Due to transmission-line theory ${ }^{91}$ and the quarterwavelength separation of the aluminum ground plane from the ITO layer, the former acts an open circuit at the position of the latter. It is in this way that matching the impedance of ITO layer to free-space is sufficient to achieve perfect absorption. However, in the case of our metasurface OSR, $\mathrm{SiO}_{2}$ has a strong dispersion in the thermal-infrared due to phonon vibrations around 10 and $20 \mu \mathrm{m}$, as visible in Figure S2. This strong dispersion in $n_{\mathrm{SiO}_{2}}$ means $t_{\mathrm{SiO}_{2}}=\lambda_{0} / 4 n_{\mathrm{SiO}_{2}}$ will be a greatly varying value in the thermal-infrared. Moreover, the resultant Reststrahlen bands show an increased reflectivity in the thermal-infrared, which is detrimental to $\epsilon_{\mathrm{IR}}$.

To optimize the cavity thickness based on these considerations, we kept $P$ at $1100 \mathrm{~nm}, h$ at $50 \mathrm{~nm}, r$ at $500 \mathrm{~nm}$, and swept $t_{\mathrm{SiO}_{2}}$. Absorption spectrum in Figure $1 \mathrm{~h}$ clearly shows that the cavity resonances evolve to larger wavelengths as we increased $t_{\mathrm{SiO}_{2}}$. While smaller cavity thicknesses (750-1250 $\mathrm{nm}$ ) supported the resonances in the 5-10 $\mu \mathrm{m}$ range, the thicker cavities (1500-2500 nm) supported LSPRs in the 20$30 \mu \mathrm{m}$ range. Resonances around $10-15 \mu \mathrm{m}$ are supported for all of these thicknesses. To find the optimum cavity thickness, the spectral overlap of $A(\lambda, T)$ with the thermal-radiation spectrum, which peaks around $10 \mu \mathrm{m}$, is of most importance. The calculation results for the OSR parameters are outlined in Figure 1i. In Figure 1i, it can be observed that $\epsilon_{\mathrm{IR}}$ is almost constant for a wide range of $1.5-2 \mu \mathrm{m}$, while the fringes in $\alpha_{\mathrm{s}}$ is again related to the Fabry-Pérot cavity. As a result, the FoM also has fringes. Another important consideration for an OSR in space missions is its spectral response under obliquely incident radiation. To scrutinize this, we explored how Device 2 responds to obliquely incident radiation, and the results are plotted in Figure S5c-f.

Overall, this numerical analysis of Device 2 shows that it can support broad LSPRs in the thermal-infrared and reach a moderate FoM of 4.5 at the cost of requiring a throughputlimiting lithography step. Moreover, Figure $1 \mathrm{~d}$, f, and $\mathrm{h}$ all demonstrate the main limiting factor associated with Device 2 in achieving larger FoM. Device 2 has a highly ordered ITO pattern, so its diffracting/scattering and EMW trapping features are poor. This causes a significant portion of the EMWs to be reflected by $\mathrm{SiO}_{2}$ at the Reststrahlen bands. These back-reflected EMWs cannot be efficiently retrapped by the ITO layer and, consequently, they leave the device without being harnessed. In these spectral regions, the EMW-ITO interaction is inherently limited and most of the absorption is due to absorption of the EMWs in the $\mathrm{SiO}_{2}$ layer. This will be analyzed in further detail in the next part of the paper. As a result, better trapping and harvesting of this reflected radiation, while not increasing the solar absorption, is pivotal to achieve an FoM that is competitive to existing OSR solutions.

Fabrication and Characterization of the Metasurface OSR with Disordered and Densely Packed ITO Nanoforests as the Top Layer. The results of the previous subsection show that there is significant room for increasing $\epsilon_{\mathrm{IR}}$ above 0.8 , while keeping $\alpha_{\mathrm{s}}$ at a low value. This can be achieved by using a thin layer of disordered and densely packed ITO nanorod forests. For this aim, we fabricated Device 1 with the procedure outlined in the Experimental Section. The fabrication step with the most significance is realizing the top-ITO layer. To achieve it, we utilized a sputtering system but placed our samples at an oblique-angle to the ITO target. Such a deposition scheme is known as 

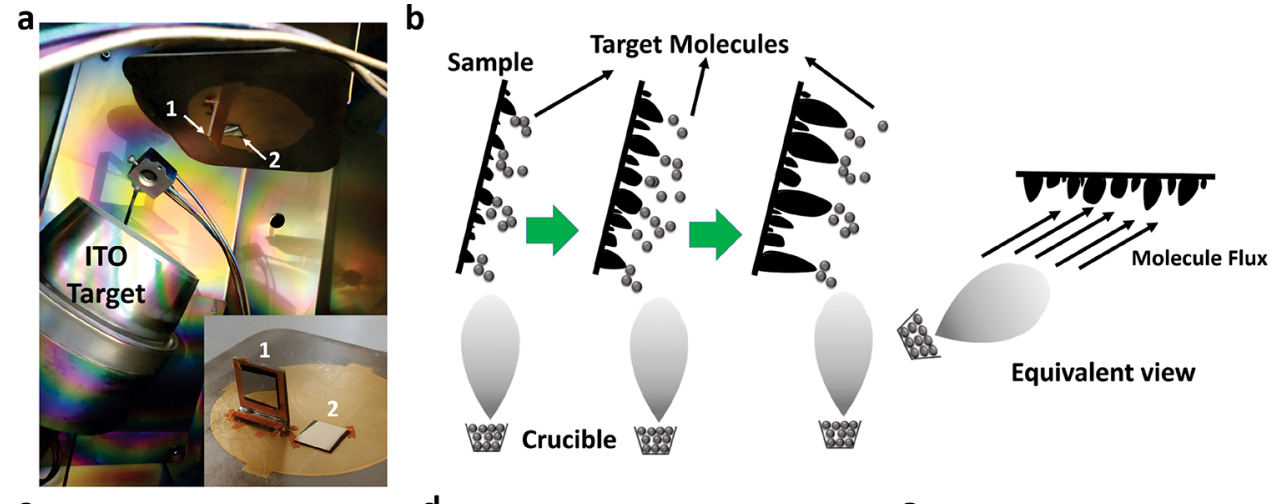

C

d
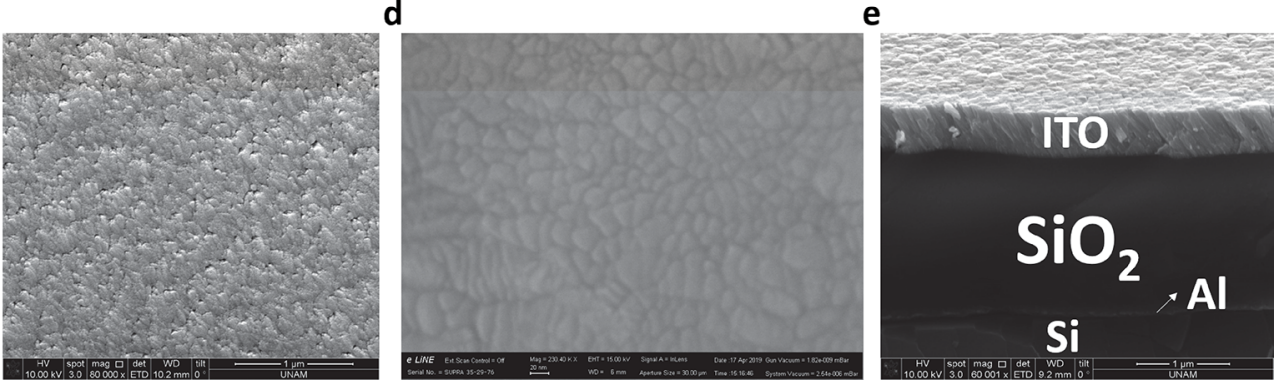

Figure 2. Fabrication of the metasurface OSR and the formation of ITO nanoforests. (a) Placement of samples in the sputtering chamber before coating of ITO. (b) Schematic illustration of line-of-sight coating in a sputtering system and the formation of tilted nanowires. (c) Low and (d) high resolution top-view SEM micrographs of one of the fabricated devices. (e) Cross-sectional SEM image of a sample metasurface OSR.
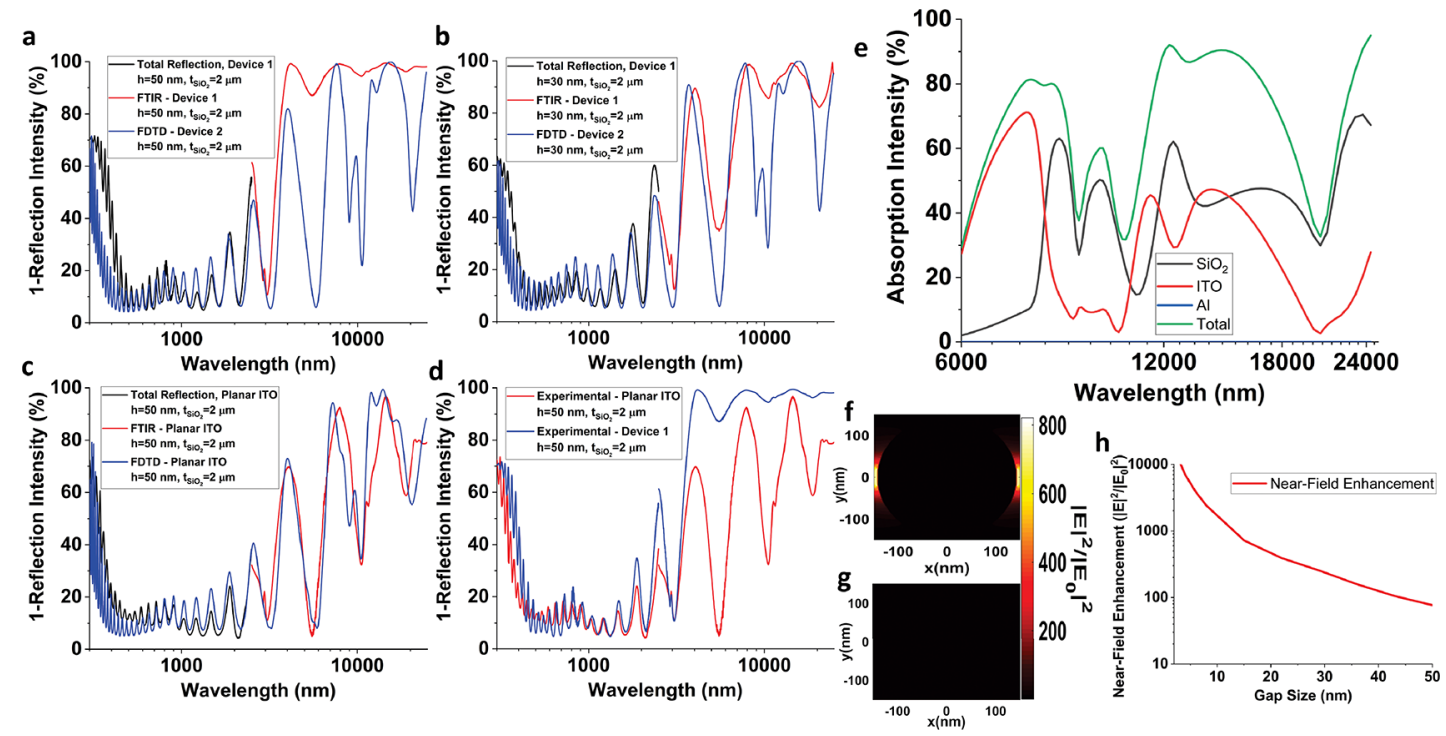

Figure 3. Optical characterization of the fabricated devices with disordered ITO nanorods, comparisons to numerical simulations of Device 2 and experimental results of a planar design, and physical explanations on $\epsilon_{\mathrm{IR}}$ enhancement in the proposed device. (a) Experimental absorption results for Device 1 with $h=50 \mathrm{~nm}$ and $t_{\mathrm{SiO}_{2}}=2 \mu \mathrm{m}$ compared to the numerical simulations of Device 2 with the same $h$ and $t_{\mathrm{SiO}_{2}}$. (b) Experimental absorption result for Device 1 with $h=30 \mathrm{~nm}$ and $t_{\mathrm{SiO}_{2}}=2 \mu \mathrm{m}$ compared to the numerical simulation Device 2 with the same $h$ and $t_{\mathrm{SiO}_{2}}$. (c) Experimental absorption result for a planar device with $h=50 \mathrm{~nm}$ and $t_{\mathrm{SiO}_{2}}=2 \mu \mathrm{m}$ compared to numerical simulations for the same device. (d) Comparison of the absorption spectra of the planar device and Device 1 with $h=50 \mathrm{~nm}$ and $t_{\mathrm{SiO}_{2}}=2 \mu \mathrm{m}$. (e) Contribution of each layer to the absorption in thermal-infrared. (f) Localization and enhancement of electric-field between nanodiscs of $P=300 \mathrm{~nm}, r=142.5 \mathrm{~nm}$. $(\mathrm{g})|E|^{2} /\left|E_{0}\right|^{2}$ value recorded at a planar ITO layer. (h) Near-field enhancement, $|E|^{2} /\left|E_{0}\right|^{2}$ values as a function of gap size between nanodiscs. In (f), (g), and (h), the $|E|^{2} /\left|E_{0}\right|^{2}$ values are recorded at $15 \mu \mathrm{m}$ and at the midgap position.

oblique-angle deposition, and it combines the inherent line-ofsight coating of PVD systems with shadowing to achieve quasi3D nanosized columnar fills with an intrinsic tilt and porosity. Figure 2a shows placement of two samples in the sputtering system. While sample 1 is placed at an oblique angle to the ITO target, sample 2 is placed in an ordinary manner for planar deposition, which is used for comparison purposes and for extracting the dielectric permittivity of ITO. The inset of Figure 2a shows the placement of samples in the sample holder plate and the special FR-4 apparatus that we designed to place the oblique-angled sample. 
In the oblique-angle deposition, a standard planar thin-film deposition, as in Sample 2, is transformed into a preferential deposition of random nucleation sites, formed during the initial stages of deposition. Continued deposition results in the deposition on these nucleation sites, while no deposition takes place in the shadowed regions. Consequently, disordered and densely packed nanocolumns emerge and they are intrinsically tilted toward the source. Such a process flow, along with an equivalent picture, is illustrated schematically in Figure $2 \mathrm{~b}$. The formation of such nanorod forests is verified by using planar and cross-sectional scanning electron microscopy (SEM) micrographs, as presented in Figure $2 \mathrm{c}$, $\mathrm{d}$, and e, respectively. The device in these figures has a $400 \mathrm{~nm}$ coating of ITO (which is larger than the devices to be mentioned later in this paper) in order to better demonstrate the details of the nanoforests, as well as the tilting of nanowires toward the source.

In finding the reflection spectrum of the fabricated devices, measurements in the UV/VIS/NIR ranges are carried out in a total reflection spectrophotometer. In the thermal-infrared, the Fourier Transform Infrared Spectroscopy (FTIR) technique is utilized, and the details for optical characterization are given in the Experimental Section. The absorption spectra for the fabricated devices are then found by $A=1-R$.

In our initial set of fabrication and experiments, we fabricated Device 1 with the thickness of the ITO layer as $50 \mathrm{~nm}$ and the $\mathrm{SiO}_{2}$ thickness as $2 \mu \mathrm{m}$. The experimental absorption spectrum of this device, in comparison to the FDTD simulations of Device 2 using periodic discs of $50 \mathrm{~nm}$ thickness, is presented in Figure 3a. In the FDTD simulations, the periodicity of the discs is set as $1100 \mathrm{~nm}$ and its diameter is $1 \mu \mathrm{m}$. The result in Figure 3a demonstrates a remarkable enhancement of light-matter interaction in Device 1 compared to Device 2 and, consequently, ultrabroadbandabsorption in the thermal-infrared, resulting in an outstanding $\epsilon_{\mathrm{IR}}$ of 0.962 .

From the space missions perspective, it is equally important to keep $\alpha_{\mathrm{s}}$ within acceptable limits while increasing $\epsilon_{\mathrm{IR}}$. It is critical to mention that our designed scaffold with high diffraction/scattering and light trapping also means solar radiation interacts more with the low-loss dielectric ITO, which may result in higher $\alpha_{\mathrm{s}}$. To alleviate this, a thin ITO layer is needed to simultaneously provide a large $\epsilon_{\mathrm{IR}}$, while not emphasizing losses in the solar spectrum. In Figure 3a, it is seen that Devices 1 and 2 produce similar absorption profiles in the solar spectrum below the bandgap of ITO, where the losses due to ITO are not significant. However, below $400 \mathrm{~nm}$, interband transitions of ITO become a prominent loss mechanism, and strong light-matter interactions in the Device 1 produce more absorption than Device 2. Nevertheless, using only $50 \mathrm{~nm}$ ITO thickness allowed us to keep $\alpha_{\mathrm{s}}$ at 0.168 . The FoM reached a record-high value of 5.73. Another important observation from Figure $3 \mathrm{a}$ is that the spectral positions of the peaks and dips in the absorption spectrum have great agreement between the FDTD simulations of Device 2 and the experimental result of Device 1 . This is because these peaks and dips are determined by the phase accumulation during the round-trip in the $\mathrm{SiO}_{2}$ cavity and the added phases due to reflections at $\mathrm{ITO}-\mathrm{SiO}_{2}$ and $\mathrm{SiO}_{2}-\mathrm{Al}$ boundaries, as given in eq S3.

In an attempt to further improve the FoM, it is more important to decrease $\alpha_{\mathrm{s}}$ rather than increasing $\epsilon_{\mathrm{IR}}$ as the former is already very close to unity in Device 1 , with $h=50$ $\mathrm{nm}$ and $t_{\mathrm{SiO}_{2}}=2 \mu \mathrm{m}$. Moreover, the numerical results for Device 2 show that thicker layers of ITO accentuate $\alpha_{\mathrm{s}}$ more than $\epsilon_{\mathrm{IR}}$, so FoM is deteriorated. Therefore, in our second set of experiments, thicker layers of ITO are not investigated, and we reduced the thickness of the ITO layer to $30 \mathrm{~nm}$. The experimental absorption spectrum of this device is shown in Figure $3 b$, along with the spectrum for Device 2, while the other geometrical parameters are the same as before. In the solar spectrum, the intention of decreasing $\alpha_{\mathrm{s}}$ is accomplished, as it decreased to 0.161 due to the smaller thickness of ITO. In the thermal-infrared, however, Device 1 , with $h=30 \mathrm{~nm}$, has worse absorption when compared to the $h=50 \mathrm{~nm}$ case, due to the poorer light-trapping capability of the scaffold. Similar to the $h=50 \mathrm{~nm}$ case, Device 1 still outperforms Device 2 in the $h=30 \mathrm{~nm}$ case, as $\epsilon_{\mathrm{IR}}$ reached 0.884 and the FoM is 5.49.

We also compared Device 1 with another lithography-free design that utilizes a planar ITO layer to further represent the power of the designed ITO nanoforests as a light-trapping scaffold. The experimental result for the planar device with $h=$ $50 \mathrm{~nm}$ and $t_{\mathrm{SiO}_{2}}=2 \mu \mathrm{m}$ is first shown in Figure $3 \mathrm{c}$, as well as the FDTD simulations for this device. In Figure 3d, we compare the experimental results for Device 1 and the planar design with the $h=50 \mathrm{~nm}$ and $t_{\mathrm{SiO}_{2}}=2 \mu \mathrm{m}$ parameters. While the planar design achieves an $\alpha_{\mathrm{s}}$ of 0.151 , its $\epsilon_{\mathrm{IR}}$ is only 0.697 , so the FoM is limited to 4.61. The planar design gives the worst $\epsilon_{\mathrm{IR}}$, because it lacks both the light-trapping capability of Device 1 and the LSPRs supported in Devices 1 and 2 . However, it does not suffer from diffraction/scattering and the resultant excited guided-modes of the structure. ${ }^{92}$ Overall, the ITO nanoforest design still outweighs the planar design.

Our proposed device is experimentally confirmed to provide an excellent scaffold for ultrabroadband thermal-infrared absorption and provides far superior $\epsilon_{\mathrm{IR}}$ compared to Device 2. There are five underlying physical reasons for this. First and foremost, the strong dispersion (see Figure S4b) and phonon reflection of $\mathrm{SiO}_{2}$ at the Reststrahlen bands was the primary reason for limiting $\epsilon_{\mathrm{IR}}$ of Device 2 under 0.8 . In those regions, incident radiation is back-reflected, and a significantly smaller portion of the radiation is trapped and harvested in the device. To make matters worse, the back-reflected EMWs cannot be efficiently retrapped in Device 2 by the periodic ITO nanodiscs, due to their poor diffraction/scattering and trapping features. Therefore, these back-reflected EMWs are not harnessed in Device 2, and light-ITO interaction is limited in these areas. This is analyzed in more detail and verified numerically by placing three three-dimensional (3D) monitors on the $\mathrm{Al}, \mathrm{SiO}_{2}$, and ITO layers and calculating the contribution of each layer to absorption in the thermalinfrared. We used the formula in eq 3 ,

$$
\frac{\mathrm{d} P_{\text {loss }}}{\mathrm{d} V}=\frac{1}{2} w \epsilon^{\prime \prime}(w)|E|^{2}
$$

where $P_{\text {loss }}$ is the absorbed power due to conduction losses and dielectric hysteresis, $V$ is the volume, $w$ is the angular frequency, $\epsilon^{\prime \prime}(w)$ is the imaginary part of the complex permittivity, and $|E|^{2}$ is the modulus of the complex electricfield inside each layer. ${ }^{93} \mathrm{~d} P_{\text {loss }} / \mathrm{d} V$ is integrated over each layer and normalized to the incident power to obtain absorption of each layer.

The absorption spectrum in Figure $3 \mathrm{e}$ clearly shows that in the phonon reflection bands (around 10 and $20 \mu \mathrm{m}$ ) of the $\mathrm{SiO}_{2}$ absorption of the transmitted light in the cavity is the 

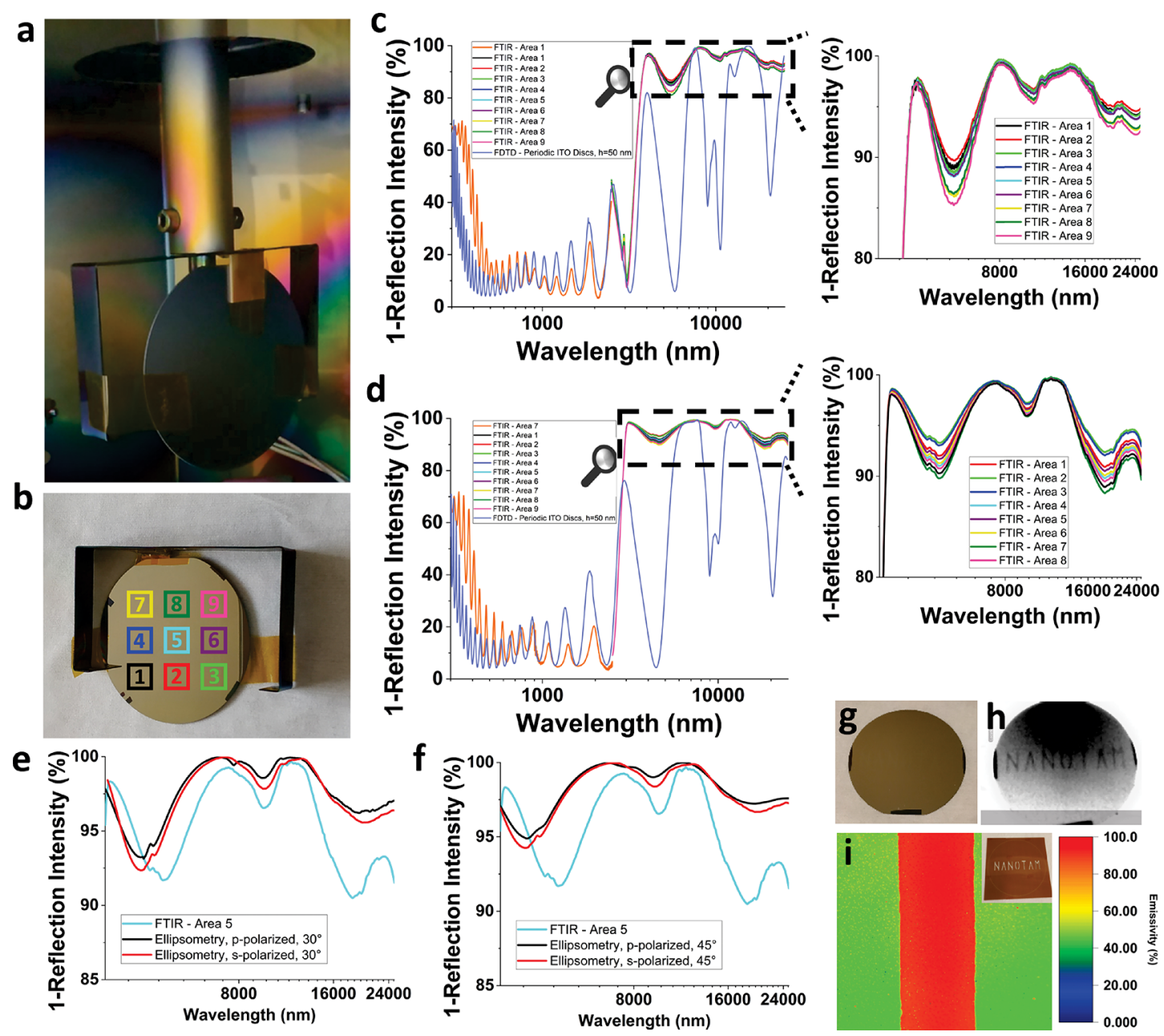

Figure 4. Large-scale fabrication and optical characterization of the proposed device. (a) Positioning of the wafer in the sample holder as seen from the back view. (b) Measurement areas in the fabricated wafers. Experimental absorption result for Device 1 with $h=50 \mathrm{~nm}$ and $(\mathrm{c}) t_{\mathrm{SiO}_{2}}=2 \mu \mathrm{m}$ and (d) $t_{\mathrm{SiO}_{2}}=1.55 \mu \mathrm{m}$, when fabricated on a wafer. Absorption spectrum under obliquely incident radiation at an incidence angle of (e) $30^{\circ}$ and (g) $45^{\circ}$. Device 1 , as seen with the $(\mathrm{g})$ naked eye and by $(\mathrm{h})$ thermal camera. (i) Thermal microscope image of Device 1 . The ellipsometry measurements are taken from area 5 .

main driver behind the absorption spectrum. However, this strong reflection is mitigated in Device 1, which is clearly visible upon comparing the absorption spectra of Devices 1 and 2 around 10 and $20 \mu \mathrm{m}$ in Figure 3a. This is a direct result of the rough and highly diffracting/scattering top-layer ITO scaffold. While the thermal radiation is reflected in Device 2 at Reststrahlen bands, in Device 1, the scaffold traps this thermal radiation (or, in other words, the residing time of thermal radiation in the structure is improved) and it is absorbed by the hybrid system of broad plasmonic resonances supported by ITO and phonon bands of $\mathrm{SiO}_{2}$. The fact that thinner ITO layers suffer more from reflection losses (by comparing Figure $3 \mathrm{a}$ and $\mathrm{b}$ ) also supports the argument that increasing the residing time of EMWs in Device 1 and coupling them to the ITO/ $\mathrm{SiO}_{2}$ hybrid is the main driver in enhanced absorption.

Second, ITO offers a plasmonic response with relatively high losses, ${ }^{71}$ so the excited LSPRs inherently have a large line width. Third, the randomness in the morphology of the nanorods, as confirmed in the SEM images in Figure 2d,e, results in multiple adjacent LSPRs of different strength, spectral position, and line width. Since our fabrication route generates random geometries, the superposition of the excited LSRPS then contribute to a flat, perfect absorption over an ultrabroad bandwidth. Fourth, the gap distances between the tilted nanorods is found to be very small and not uniform. It is shown in the literature that extremely small gaps between plasmonic nanostructures result in the extreme enhancement and localization of optical fields. ${ }^{94}$ We show the near-field enhancement of optical fields between the ITO nanodiscs, due to the dipolar LSPRs in Figure 3f, as an exemplary case from Device 2. The optical field inside the ITO layer of a planar device is also calculated, and the result is shown in Figure $3 \mathrm{~g}$. Comparison of Figure $3 \mathrm{f}$ and $\mathrm{g}$ demonstrates that the plasmonic resonance and the corresponding electromagnetic hotspots between the nanodiscs do not exist in the planar case. This is the reason why the planar design had the poorest $\epsilon_{\mathrm{IR}}$. In Figure $3 \mathrm{~h}$, we indicate that further decreasing the gap distance between the nanodiscs results in higher enhancement of optical fields. This extreme localization results in larger optical losses, as a result of eq 3. Thus, the formation of deep subwavelength gaps between the nanowires, as demonstrated in Figure 2d,e, triggers the existence of intense local fields, and these hotspots lead to strong light absorption in the thermal infrared. Moreover, when the distance between the nanostructures become very small, the position of plasmon resonances is determined by the gap size, rather than the individual 
dimensions of the nanostructures. The randomness in the porosity of the deposited film, as shown in Figure 2c, is then a contributor to the perfect absorption.

Lastly, the random nanorods in Device 1 diffract light in more directions, compared to the periodic discs of Device 2. Therefore, Device 1 may be aiding the coupling of a larger portion of incident radiation to the surface-plasmon polaritons (SPPs) ${ }^{88}$ in the $\mathrm{SiO}_{2}-\mathrm{Al}$ interface.

Large-Scale Fabrication of the Proposed Device and Optical Characterization. Oblique-angle deposition not only offers a substantial enhancement to the OSR performance, it also enables a facile route for large-scale fabrication, which is of paramount importance to establish a competitive OSR technology to the planar conventional devices. As a proof-of-concept, Device 1 with $h=50 \mathrm{~nm}$ and $t_{\mathrm{SiO}_{2}}=2 \mu \mathrm{m}$ is fabricated on a 4-in. silicon wafer. The wafer is placed in the sputtering chamber at an angle to the source, similar to Figure 2a. To do that, the plate is removed, and the wafer is directly fixed in the sample holder, as shown in Figure 4a, which shows the attachment of wafer from the back side. The same apparatus used for small area samples is also used to put the wafer at an oblique angle, and two strips are adhered to the sides of wafer for additional support. The wafer is characterized from nine different areas, which are shown in Figure $4 b$ to indicate the adaptation of oblique-angle deposition technique to large-scale fabrication, and also examine the uniformity of the coating. The experimental results are shown in Figure 4c. The solar absorption is measured only from area 5 and $\alpha_{\mathrm{s}}$ is calculated as 0.167 , which is almost identical to the small-area fabrication. On the other hand, spectral response in thermalinfrared is measured from all nine areas. To further discern the variations in the absorption spectra between different areas, we zoomed into $3 \mu \mathrm{m}$ to $25 \mu \mathrm{m}$ region. It is seen that the regions further away from the source has slightly lower absorption, which is attributed to the minor nonuniformity in ITO thickness within the wafer. Nevertheless, the smallest $\epsilon_{\mathrm{IR}}$ is found to be 0.957 , while its highest value reached 0.97 , so the variations within the wafer are not significant.

Experimental results up to now have demonstrated the optimized optical performance of the metasurface OSRs. Nevertheless, decreasing the thickness of $\mathrm{SiO}_{2}$ is still desirable to decrease the launch and assembly costs and the weight of the OSRs when they are used to coat a spacecraft. The numerical results of Device 2 has shown that several different cavity thicknesses can result in almost equal FoM. It can be seen in Figure 1i that a cavity thickness between 1.5 and 1.6 $\mu \mathrm{m}$ gives the peak FoM, while also being the most tolerant area to thickness inaccuracies. Device 1 with $h=50 \mathrm{~nm}$ and $t_{\mathrm{SiO}_{2}}=$ $1.55 \mu \mathrm{m}$ is fabricated on a 4-in. wafer and is characterized in the same way as before. Experimental result for this device and numerical result for Device 2 with the same $h$ and $t_{\mathrm{SiO}_{2}}$ are shown in Figure 4d. The experimental results show similar characteristics to Figure $4 c$, and the physical reasoning outlined until now applies to this sample. The maximum $\epsilon_{\mathrm{IR}}$ is calculated as 0.968 , while its minimum value is $0.952 . \alpha_{\mathrm{s}}$ is found to be 0.169 . Therefore, almost identical performance to the ITOD50CAV2000 device is achieved with a smaller thickness of $\mathrm{SiO}_{2}$.

Spectral response of Device 1 with $h=50 \mathrm{~nm}$ and $t_{\mathrm{SiO}_{2}}=$ $1.55 \mu \mathrm{m}$ under obliquely incident radiation is measured experimentally in the thermal-infrared, from 3 to $25 \mu \mathrm{m}$, by utilizing ellipsometry method. The results at $30^{\circ}$ and $45^{\circ}$ incidence angles are shown in Figure $4 \mathrm{e}$ and $\mathrm{f}$, respectively, for both $\mathrm{p}$ - and s-polarized incident radiations. The blue-shifting of the spectral response as well as increased absorption behavior are observed in these results, which are in harmony with the numerical results for Device 2, given in Figure S5e.

In this paper, up to this point, we have demonstrated that the outstanding performance of our proposed device fulfills the key functionality of an OSR, that is, a very high $\epsilon_{\mathrm{IR}}$, a small $\alpha_{\mathrm{s}}$, large-scale compatibility, and uniformity. Our proposed device has a thickness around $2 \mu \mathrm{m}$, which is at least 2 orders of magnitude smaller than that of the conventional OSRs. Nevertheless, application of these devices to the radiative cooling of spacecraft in real-time necessitates further studies in scrutinizing the heat transfer and finding the cooling rates. In addition to these, the ongoing studies will focus on applying these ideas to flexible substrates and tests involving the mechanical, thermal, UV, and radiation resistance of the proposed devices. The perfect absorption in the thermalinfrared range and the experimentally obtained $\epsilon_{\mathrm{IR}}$ of 0.968 are also very promising in the areas of thermal-imaging cameras with labeled security. As a proof-of-concept, a mask with drilled holes to create the labeling word "NANOTAM" is placed on the wafer before sputtering. After fabricating Device 1 with $h=50 \mathrm{~nm}$ and $t_{\mathrm{SiO}_{2}}=1.55 \mu \mathrm{m}$, the wafer is observed with a thermal camera. Lithography is not preferred to create the label in order not to sacrifice from large-scale compatibility. The view of the wafer with the naked eye is given in Figure $4 \mathrm{~g}$, and the "NANOTAM" label is barely recognizable. However, when this wafer is inspected under a thermal camera, the large emissivity difference between the ITO-coated versus noncoated areas makes the label clearly discernible, as shown in Figure $4 \mathrm{~h}$. In Figure $4 \mathrm{i}$, we show a thermal microscope micrograph from one of the areas. The sharp contrast between the ITO-coated areas (appear red due to very high thermalemissivity) versus the noncoated areas is clearly distinguishable. It is also important to note from Figure $4 \mathrm{i}$ that the areas with only $\mathrm{SiO}_{2}$ coating still have an $\epsilon_{\mathrm{IR}}$ of 0.5 . This further supports our argument that the perfect absorption in the proposed devices are a result of the hybrid system of ITO and $\mathrm{SiO}_{2}$. Last but not least, the very high performance of our proposed devices can also find applications in daytime radiative coolers. $^{40,41,43}$ However, the overall flat absorption over the entire thermal-infrared should be adapted to the atmospheric absorption lines, which is an ongoing study at this time.

\section{CONCLUSIONS}

In conclusion, metasurfaces utilizing disordered, densely packed, and titled ITO nanorod forests as the top absorbing layer are experimentally analyzed in this paper and the attainment of a record-high thermal-emissivity of 0.968 , and Figure of Merit of 5.73 are verified. The top ITO scaffold of our metasurfaces enabled the very efficient harvesting of thermal-radiation to have an ultrabroadband absorption in the thermal-infrared range, while their small thickness did not accentuate the dielectric losses in the solar spectrum. The utilization of oblique-angle deposition with the line-of-sight coating of PVD systems allowed not only the realization of such ITO nanoforests, but also large-scale and high-throughput fabrication. The experimental results of the proposed devices are compared to the numerical simulations of periodic ITO discs and the experimental results of the planar ITO thin-film as the top absorbing layer. These comparisons further proved 
the absorption enhancement by the ITO nanoforests, which is mainly due to relieving the strong phonon reflection bands of $\mathrm{SiO}_{2}$ in thermal-infrared. Overall, our ultrahigh performance, yet large-scale compatible devices hold great promise for the radiative cooling of spacecraft during their space missions, and it is also highly applicable to many areas such as terrestrial radiative coolers and thermal imaging for labeled security purposes.

\section{EXPERIMENTAL SECTION}

Fabrication of the Metasurface OSR. For the fabrication of the small-area samples, first, a typical commercial squareshaped silicon substrate with a $225 \mathrm{~mm}^{2}$ area was cleaned with acetone, isopropanol (IPA), and deionized water and then dried with a $\mathrm{N}_{2}$ flow, to be used as a substrate. The surface was further treated using oxygen plasma for 20 min before starting the deposition of thin films. Same procedure is followed for the 4-in. wafers. First, $125 \mathrm{~nm}$ thick aluminum back-reflector is coated by using an electron-beam evaporator system (Leybold Univex 350) at a chamber pressure of 3e-6 to $5 \mathrm{e}-6 \mathrm{mbar}$ and at a rate of $2 \AA /$ s. Next, a $\mathrm{SiO}_{2}$ layer with the thicknesses mentioned in the main text are coated by using a PlasmaEnhanced Chemical Vapor Deposition (PECVD) system (SAMCO). This process is carried out with $\mathrm{SiH}_{4}$ and $\mathrm{N}_{2} \mathrm{O}$ flow rates of $300 \mathrm{sccm}$ at a temperature of $350{ }^{\circ} \mathrm{C}$. Finally, an ITO layer is coated in an RF-magnetron sputtering system (Leybold Univex 350) at a processing pressure of 2.2e-3 mbar, RF power of $125 \mathrm{~W}$, and a rate of $1.3 \AA / \mathrm{s}$. The flow rate of high purity (99.999\%) sputtering Argon (Ar) gas, free of oxygen, was $7 \mathrm{sccm}$ during the process. No oxygen was added during the deposition. The sample holder is not rotated during the process.

Optical Characterization. To measure the thermal emissivity, $\epsilon_{\mathrm{IR}}$ of the samples with disordered ITO nanoforests and planar ITO thin film structures, reflectance in the thermalinfrared spectrum was measured over the range of 2.5 to $25 \mu \mathrm{m}$ using an FTIR microscope with a numerical aperture of 0.4 (Bruker-Vertex 70v, Hyperion microscope). The instrument employs a mid-infrared source, $\mathrm{KBr}$ beamsplitter and nitrogencooled Mercury Cadmium Telluride (MCT-D313) detector. A $125 \mathrm{~nm}$ aluminum coated $\mathrm{SiO}_{2} / \mathrm{Si}$ substrate was used as reference. Solar absorptance of all the samples are obtained by measuring the UV/VIS/NIR reflectance over the range from $300 \mathrm{~nm}$ to $2.5 \mu \mathrm{m}$. In these measurements, a commercial total reflection spectrophotometer (Agilent Cary 5000), utilizing a diffuse reflectance accessory (DRA) of a diameter of $110 \mathrm{~mm}$ is used. White polytetrafluoroethylene (PTFE) is used as a reference. Oblique-angle measurements in the thermal-infrared are carried out by using a J. A. Woollam Co. Inc. IR-VASE Mark II ellipsometer. The emissivity difference between ITOcoated versus noncoated areas are investigated by using a commercial thermal camera (Seek Thermal). The microscopy images for the same purpose are taken by a nitrogen-cooled InfraScope MWIR Temperature Mapping Microscope from Quantum Focus Instruments (QFI) Corp.

Numerical Simulations. In the FDTD simulations, we illuminated the unit cell with a broadband plane wave at normal incidence whose electric-field is polarized in the $x$ direction. In the $x$ - and $y$-directions of the simulation region, we used periodic boundary conditions, while in the $z$-direction perfectly matched layers (PML) are adopted and they are placed four wavelengths away from the structure. We used a single monitor that was at an adequate distance away from the structure to collect the reflected EMWs and calculate the reflected $(R)$ power, which is normalized to the incident power. Due to the fact that the bottom $\mathrm{Al}$ thickness was chosen to be much larger than the skin-depth of EMWs at the spectral regions of interest, the transmitted power $(T)$ was considered to be negligible. After verifying this in the simulations, we calculated the spectral absorption by using $A$ $=1-R$. To be able to increase the stability of the simulations and spectral resolution of the absorption spectrum, all of the FDTD simulations were divided into three regions: wavelength from $300 \mathrm{~nm}$ to $2.5 \mu \mathrm{m}, 2.5$ to $15 \mu \mathrm{m}$, and 15 to $30 \mu \mathrm{m}$.

\section{ASSOCIATED CONTENT}

\section{S Supporting Information}

The Supporting Information is available free of charge on the ACS Publications website at DOI: 10.1021/acsphotonics.9b00636.

Numerical simulation results, including the comparison of different mirror and spacer materials on the OSR performance, effect of unit-cell periodicity on the spectral response and OSR parameters, and performance under obliquely incident radiation (PDF)

\section{AUTHOR INFORMATION}

\section{Corresponding Authors}

*E-mail: yildirim@ee.bilkent.edu.tr.

*E-mail: ozbay@bilkent.edu.tr.

ORCID $\odot$

Deniz Umut Yildirim: 0000-0002-2160-1142

Notes

The authors declare no competing financial interest.

\section{ACKNOWLEDGMENTS}

Authors acknowledge financial support from the Scientific and Technological Research Council of Turkey (TUBITAK) and DPT-HAMIT under Project Nos. 113E331, 114E374, and 115F560. One of the authors (E.O.) also acknowledges partial support from the Turkish Academy of Sciences (TUBA).

\section{REFERENCES}

(1) Pisacane, V. In Fundamentals of Space Systems; Vincent, L., Ed.; Oxford University Press, 2005; p 848.

(2) Ovchinnikov, M.; Mckenna-lawlor, S.; Psychology, S.; Kanas, N.; Manuy, D.; Larson, W. J.; Wertz, J. R. Space Mission Analysis and Design; Microcosm, 1991.

(3) Gilmore, D. The Aerospace Press; American Institute of Aeronautics and Astronautics, Inc., 2002; p 1.

(4) BREUCH, R. A.; MARSHALL, K. N. Optical solar reflector - A highly stable, low alpha sub $S$ /epsilon spacecraft thermal control surface. J. Spacecr. Rockets 1968, 5, 1051-1056.

(5) Sun, K.; Riedel, C. A.; Wang, Y.; Urbani, A.; Simeoni, M.; Mengali, S.; Zalkovskij, M.; Bilenberg, B.; De Groot, C. H.; Muskens, O. L. Metasurface Optical Solar Reflectors Using AZO Transparent Conducting Oxides for Radiative Cooling of Spacecraft. ACS Photonics 2018, 5, 495-501.

(6) Sun, K.; Riedel, C. A.; Urbani, A.; Simeoni, M.; Mengali, S.; Zalkovskij, M.; Bilenberg, B.; De Groot, C. H.; Muskens, O. L. VO2 Thermochromic Metamaterial-Based Smart Optical Solar Reflector. ACS Photonics 2018, 5, 2280-2286.

(7) Hoffman, A. J.; Alekseyev, L.; Howard, S. S.; Franz, K. J.; Wasserman, D.; Podolskiy, V. A.; Narimanov, E. E.; Sivco, D. L.; Gmachl, C. Negative refraction in semiconductor metamaterials. Nature Mater. 2007, 6, 946. 
(8) Smith, D. R.; Pendry, J. B.; Wiltshire, M. C. Metamaterials and negative refractive index. Science 2004, 305, 788.

(9) Lapine, M.; Shadrivov, I. V.; Powell, D. A.; Kivshar, Y. S. Magnetoelastic metamaterials. Nat. Mater. 2012, 11, 30.

(10) Papadakis, G. T.; Fleischman, D.; Davoyan, A.; Yeh, P.; Atwater, H. A. Optical magnetism in planar metamaterial heterostructures. Nat. Commun. 2018, 9, na.

(11) Li, Z.; Mutlu, M.; Ozbay, E. Chiral metamaterials: From optical activity and negative refractive index to asymmetric transmission. $J$. Opt2013.15023001

(12) Menzel, C.; Helgert, C.; Rockstuhl, C.; Kley, E. B.; Tünnermann, A.; Pertsch, T.; Lederer, F. Asymmetric transmission of linearly polarized light at optical metamaterials. Phys. Rev. Lett. 2010, 104, na.

(13) Valentine, J.; Li, J.; Zentgraf, T.; Bartal, G.; Zhang, X. An optical cloak made of dielectrics. Nat. Mater. 2009, 8, 568.

(14) Schurig, D.; Mock, J. J.; Justice, B. J.; Cummer, S. A.; Pendry, J. B.; Starr, A. F.; Smith, D. R. Metamaterial electromagnetic cloak at microwave frequencies. Science 2006, 314, 977.

(15) Fang, A.; Koschny, T.; Soukoulis, C. M. Lasing in metamaterial nanostructures. Journal of Optics A: Pure and Applied Optics 2010, 12, 024013.

(16) Plum, E.; Fedotov, V. A.; Kuo, P.; Tsai, D. P.; Zheludev, N. I. Towards the lasing spaser: controlling metamaterial optical response with semiconductor quantum dots. Opt. Express 2009, 17, 8548.

(17) Zheludev, N. I.; Kivshar, Y. S. From metamaterials to metadevices. Nature Materials 2012, 11, 917-924.

(18) Yu, N.; Capasso, F. Flat optics with designer metasurfaces. Nature Materials 2014, 13, 139-150.

(19) Neshev, D.; Aharonovich, I. Optical metasurfaces: new generation building blocks for multi-functional optics. Light: Science and Applications 2018, 7, na.

(20) Yu, N.; Genevet, P.; Kats, M. A.; Aieta, F.; Tetienne, J. P.; Capasso, F.; Gaburro, Z. Light propagation with phase discontinuities: Generalized laws of reflection and refraction. Science 2011, 334, 333.

(21) Kuznetsov, A. I.; Miroshnichenko, A. E.; Brongersma, M. L.; Kivshar, Y. S.; Luk'yanchuk, B. Optically resonant dielectric nanostructures. Science 2016, 354, 6314.

(22) Khorasaninejad, M.; Chen, W. T.; Devlin, R. C.; Oh, J.; Zhu, A. Y.; Capasso, F. Metalenses at visible wavelengths: Diffraction-limited focusing and subwavelength resolution imaging. Science 2016, 352, 1190.

(23) Wang, S.; et al. A broadband achromatic metalens in the visible. Nat. Nanotechnol. 2018, 13, 227.

(24) Hsiao, H.-H.; Chu, C. H.; Tsai, D. P. Fundamentals and Applications of Metasurfaces. Small Methods 2017, 1, na.

(25) Watts, C. M.; Liu, X.; Padilla, W. J. Metamaterial electromagnetic wave absorbers. Adv. Mater. 2012, 24, na.

(26) Landy, N. I.; Sajuyigbe, S.; Mock, J. J.; Smith, D. R.; Padilla, W. J. Perfect metamaterial absorber. Phys. Rev. Lett. 2008, 100, na.

(27) Ellenbogen, T.; Seo, K.; Crozier, K. B. Chromatic plasmonic polarizers for active visible color filtering and polarimetry. Nano Lett. 2012, 12, 1026

(28) Ji, C.; Lee, K. T.; Xu, T.; Zhou, J.; Park, H. J.; Guo, L. J. Engineering Light at the Nanoscale: Structural Color Filters and Broadband Perfect Absorbers. Advanced Optical Materials 2017, 5 (20), 1700368.

(29) Landy, N. I.; Bingham, C. M.; Tyler, T.; Jokerst, N.; Smith, D. R.; Padilla, W. J. Design, theory, and measurement of a polarizationinsensitive absorber for terahertz imaging. Physical Review B Condensed Matter and Materials Physics 2009, 79, na.

(30) Offermans, P.; Schaafsma, M. C.; Rodriguez, S. R.; Zhang, Y.; Crego-Calama, M.; Brongersma, S. H.; Gómez Rivas, J. Universal scaling of the figure of merit of plasmonic sensors. ACS Nano 2011, 5, 5151.

(31) Stewart, M. E.; Anderton, C. R.; Thompson, L. B.; Maria, J.; Gray, S. K.; Rogers, J. A.; Nuzzo, R. G. Nanostructured plasmonic sensors. Chem. Rev. 2008, 108, 494-521.
(32) Tittl, A.; Michel, A. K. U.; Schäferling, M.; Yin, X.; Gholipour, B.; Cui, L.; Wuttig, M.; Taubner, T.; Neubrech, F.; Giessen, H. A Switchable Mid-Infrared Plasmonic Perfect Absorber with Multispectral Thermal Imaging Capability. Adv. Mater. 2015, 27, 4526.

(33) Xu, T.; Wu, Y.-K.; Luo, X.; Guo, L. J. Plasmonic nanoresonators for high-resolution colour filtering and spectral imaging. Nat. Commun. 2010, 1, na.

(34) Yildirim, D. U.; Ghobadi, A.; Ozbay, E. Near-absolute polarization insensitivity in grapheme based ultra-narrowband perfect visible light absorber. Sci. Rep. 2018, 8, 1-14.

(35) Yokogawa, S.; Burgos, S. P.; Atwater, H. A. Plasmonic color filters for CMOS image sensor applications. Nano Lett. 2012, 12, 4349.

(36) Rephaeli, E.; Fan, S. Absorber and emitter for solar thermophotovoltaic systems to achieve efficiency exceeding the ShockleyQueisser limit. Opt. Express 2009, 17, 15145.

(37) Tong, J. K.; Hsu, W. C.; Huang, Y.; Boriskina, S. V.; Chen, G. Thin-film 'thermal well' emitters and absorbers for high-efficiency thermophotovoltaics. Sci. Rep. 2015, 5, na.

(38) García De Arquer, F. P.; Mihi, A.; Konstantatos, G. Large-Area Plasmonic-Crystal-Hot-Electron-Based Photodetectors. ACS Photonics 2015, 2, 950.

(39) Li, W.; Valentine, J. Metamaterial perfect absorber based hot electron photodetection. Nano Lett. 2014, 14, 3510.

(40) Lu, Y.; Chen, Z.; Ai, L.; Zhang, X.; Zhang, J.; Li, J.; Wang, W.; Tan, R.; Dai, N.; Song, W. A Universal Route to Realize Radiative Cooling and Light Management in Photovoltaic Modules. Solar RRL 2017, 1, 1700084.

(41) Ko, B.; Lee, D.; Badloe, T.; Rho, J. Metamaterial-Based Radiative Cooling: Towards Energy-Free All-Day Cooling. Energies 2019, 12, 89.

(42) Zhao, B.; Hu, M.; Ao, X.; Chen, N.; Pei, G. Radiative cooling: A review of fundamentals, materials, applications, and prospects. Appl. Energy 2019, 236, 489-513.

(43) Zhai, Y.; Ma, Y.; David, S. N.; Zhao, D.; Lou, R.; Tan, G.; Yang, R.; Yin, X. Scalable-manufactured randomized glass-polymer hybrid metamaterial for daytime radiative cooling. Science 2017, 355, 1062.

(44) Liu, T.; Takahara, J. Ultrabroadband absorber based on singlesized embedded metal-dielectric-metal structures and application of radiative cooling. Opt. Express 2017, 25, A612.

(45) Wu, D.; Liu, C.; Xu, Z.; Liu, Y.; Yu, Z.; Yu, L.; Chen, L.; Li, R.; $\mathrm{Ma}, \mathrm{R}$; Ye, H. The design of ultra-broadband selective near-perfect absorber based on photonic structures to achieve near-ideal daytime radiative cooling. Materials and Design 2018, 139, 104.

(46) Aydin, K.; Ferry, V. E.; Briggs, R. M.; Atwater, H. A. Broadband polarization-independent resonant light absorption using ultrathin plasmonic super absorbers. Nat. Commun. 2011, 2, na.

(47) Liang, Q.; Yu, W.; Zhao, W.; Wang, T.; Zhao, J.; Zhang, H.; Tao, S. Numerical study of the meta-nanopyramid array as efficient solar energy absorber. Optical Materials Express 2013, 3, 1187.

(48) Ding, F.; Dai, J.; Chen, Y.; Zhu, J.; Jin, Y.; Bozhevolnyi, S. I. Broadband near-infrared metamaterial absorbers utilizing highly lossy metals. Sci. Rep. 2016, 6, na.

(49) Nielsen, M. G.; Pors, A.; Albrektsen, O.; Bozhevolnyi, S. I. Efficient absorption of visible radiation by gap plasmon resonators. Opt. Express 2012, 20, 13311.

(50) Liang, Q.; Wang, T.; Lu, Z.; Sun, Q.; Fu, Y.; Yu, W. Metamaterial-Based Two Dimensional Plasmonic Subwavelength Structures Offer the Broadest Waveband Light Harvesting. Advanced Optical Materials 2013, 1, 43.

(51) Cui, Y.; Xu, J.; Hung Fung, K.; Jin, Y.; Kumar, A.; He, S.; Fang, N. X. A thin film broadband absorber based on multi-sized nanoantennas. Appl. Phys. Lett. 2011, 99, 253101.

(52) Feng, Q.; Pu, M.; Hu, C.; Luo, X. Engineering the dispersion of metamaterial surface for broadband infrared absorption. Opt. Lett. 2012, 37, 2133.

(53) Guo, W.; Liu, Y.; Han, T. Ultra-broadband infrared metasurface absorber. Opt. Express 2016, 24, 20586. 
(54) Wang, H.; Wang, L. Perfect selective metamaterial solar absorbers. Opt. Express 2013, 21, A1078.

(55) Bossard, J. A.; Lin, L.; Yun, S.; Liu, L.; Werner, D. H.; Mayer, T. S. Near-ideal optical metamaterial absorbers with super-octave bandwidth. ACS Nano 2014, 8, 1517.

(56) Ghobadi, A.; Hajian, H.; Gokbayrak, M.; Abedini Dereshgi, S.; Toprak, A.; Butun, B.; Ozbay, E. Visible light nearly perfect absorber: an optimum unit cell arrangement for near absolute polarization insensitivity. Opt. Express 2017, 25, 27624.

(57) Ding, F.; Jin, Y.; Li, B.; Cheng, H.; Mo, L.; He, S. Ultrabroadband strong light absorption based on thin multilayered metamaterials. Laser and Photonics Reviews 2014, 8, 946.

(58) Cui, Y.; Fung, K. H.; Xu, J.; Ma, H.; Jin, Y.; He, S.; Fang, N. X. Ultrabroadband light absorption by a sawtooth anisotropic metamaterial slab. Nano Lett. 2012, 12, 1443.

(59) Zhu, J.; Ma, Z.; Sun, W.; Ding, F.; He, Q.; Zhou, L.; Ma, Y. Ultra-broadband terahertz metamaterial absorber. Appl. Phys. Lett. 2014, 105, 021102 .

(60) Long, C.; Yin, S.; Wang, W.; Li, W.; Zhu, J.; Guan, J. Broadening the absorption bandwidth of metamaterial absorbers by transverse magnetic harmonics of 210 mode. Sci. Rep. 2016, 6, na.

(61) He, X.; Yan, S.; Lu, G.; Zhang, Q.; Wu, F.; Jiang, J. An ultrabroadband polarization-independent perfect absorber for the solar spectrum. RSC Adv. 2015, 5, 61955.

(62) Ghobadi, A.; Hajian, H.; Butun, B.; Ozbay, E. Strong LightMatter Interaction in Lithography-Free Planar Metamaterial Perfect Absorbers. ACS Photonics 2018, 5, 4203-4221.

(63) Li, Z.; Butun, S.; Aydin, K. Large-area, Lithography-free super absorbers and color filters at visible frequencies using ultrathin metallic films. ACS Photonics 2015, 2, 183.

(64) Abedini Dereshgi, S.; Ghobadi, A.; Hajian, H.; Butun, B.; Ozbay, E. Ultra-Broadband, Lithography-Free, and Large-Scale Compatible Perfect Absorbers: The Optimum Choice of Metal layers in Metal-Insulator Multilayer Stacks. Sci. Rep. 2017, 7, na.

(65) Ding, F.; Mo, L.; Zhu, J.; He, S. Lithography-free, broadband, omnidirectional, and polarization-insensitive thin optical absorber. Appl. Phys. Lett. 2015, 106, 061108.

(66) Ghobadi, A.; Dereshgi, S. A.; Hajian, H.; Birant, G.; Butun, B.; Bek, A.; Ozbay, E. 97\% light absorption in an ultrabroadband frequency range utilizing an ultrathin metal layer: Randomly oriented, densely packed dielectric nanowires as an excellent light trapping scaffold. Nanoscale 2017, 9, 16652-16660.

(67) Ghobadi, A.; Dereshgi, S. A.; Hajian, H.; Bozok, B.; Butun, B.; Ozbay, E. Ultra-broadband, wide angle absorber utilizing metal insulator multilayers stack with a multi-thickness metal surface texture. Sci. Rep. 2017, 7, na.

(68) Ghobadi, A.; Hajian, H.; Dereshgi, S. A.; Bozok, B.; Butun, B.; Ozbay, E. Disordered Nanohole Patterns in Metal-Insulator Multilayer for Ultra-broadband Light Absorption: Atomic Layer Deposition for Lithography Free Highly repeatable Large Scale Multilayer Growth. Sci. Rep. 2017, 7, na.

(69) Ghobadi, A.; Hajian, H.; Rashed, A. R.; Butun, B.; Ozbay, E. Tuning the metal filling fraction in absorbers to maximize the absorption bandwidth. Photonics Reseach 2018, 6, 168.

(70) Bae, K.; Kang, G.; Cho, S. K.; Park, W.; Kim, K.; Padilla, W. J. Flexible thin-film black gold membranes with ultrabroadband plasmonic nanofocusing for efficient solar vapour generation. Nat. Commun. 2015, 6, na.

(71) Naik, G. V.; Shalaev, V. M.; Boltasseva, A. Alternative plasmonic materials: Beyond gold and silver. Advanced Materials 2013, 25, 3264.

(72) Withnall, R. Encyclopedia of Modern Optics; Elsevier, 2005.

(73) Li, S. Q.; Guo, P.; Zhang, L.; Zhou, W.; Odom, T. W.; Seideman, T.; Ketterson, J. B.; Chang, R. P. H. Infrared plasmonics with indium-tin-oxide nanorod arrays. ACS Nano 2011, 5, 9161.

(74) Khurgin, J. B. Replacing noble metals with alternative materials in plasmonics and metamaterials: How good an idea? Philosophical Transactions of the Royal Society A: Mathematical, Physical and Engineering Sciences 2017, 375, 20160068.
(75) Kim, J.; Dutta, A.; Naik, G. V.; Giles, A. J.; Bezares, F. J.; Ellis, C. T.; Tischler, J. G.; Mahmoud, A. M.; Caglayan, H.; Glembocki, O. J.; Kildishev, A. V.; Caldwell, J. D.; Boltasseva, A.; Engheta, N. Role of epsilon-near-zero substrates in the optical response of plasmonic antennas. Optica 2016, 3, 339.

(76) Naik, G. V.; Boltasseva, A. Semiconductors for plasmonics and metamaterials. Physica Status Solidi - Rapid Research Letters 2010, 4, 295.

(77) Abb, M.; Wang, Y.; Papasimakis, N.; De Groot, C. H.; Muskens, O. L. Surface-enhanced infrared spectroscopy using metal oxide plasmonic antenna arrays. Nano Lett. 2014, 14, 346.

(78) Plummer, J. D.; Deal, M. D.; Griffin, P. B. Silicon VLSI technology: fundamentals, practice and modeling; Pearson, 2000.

(79) Barranco, A.; Borras, A.; Gonzalez-Elipe, A. R.; Palmero, A. Perspectives on oblique angle deposition of thin films: From fundamentals to devices. Progress in Materials Science 2016, 76, 59153.

(80) Zhao, Y.; Ye, D.; Wang, G.-C.; Lu, T.-M. Designing nanostructures by glancing angle deposition. Proc. SPIE 2003, 59.

(81) Wang, Z.; West, P. R.; Meng, X.; Kinsey, N.; Shalaev, V. M.; Boltasseva, A. Angled physical vapor deposition techniques for nonconformal thin films and three-dimensional structures. MRS Commun. 2016, 6, 17-22.

(82) Grüner, C.; Liedtke, S.; Bauer, J.; Mayr, S. G.; Rauschenbach, B. Morphology of Thin Films Formed by Oblique Physical Vapor Deposition. ACS Applied Nano Materials 2018, 1, 1370-1376.

(83) Mark, A. G.; Gibbs, J. G.; Lee, T. C.; Fischer, P. Hybrid nanocolloids with programmed three-dimensional shape and material composition. Nat. Mater. 2013, 12, 802.

(84) Jensen, M.; Brett, M. J. Square spiral 3D photonic bandgap crystals at telecommunications frequencies. Opt. Express 2005, 13, 3348.

(85) Lintymer, J.; Gavoille, J.; Martin, N.; Takadoum, J. Glancing angle deposition to modify microstructure and properties of sputter deposited chromium thin films. Surf. Coat. Technol. 2003, 174-175, 316

(86) Xi, J. Q.; Schubert, M. F.; Kim, J. K.; Schubert, E. F.; Chen, M.; Lin, S. Y.; Liu, W.; Smart, J. A. Optical thin-film materials with low refractive index for broadband elimination of Fresnel reflection. Nat. Photonics 2007, 1, 176.

(87) Xiao, X.; Dong, G.; Shao, J.; He, H.; Fan, Z. Optical and electrical properties of $\mathrm{SnO} 2: \mathrm{Sb}$ thin films deposited by oblique angle deposition. Appl. Surf. Sci. 2010, 256, 1636.

(88) Maier, S. A. Plasmonics: Fundamentals and Applications; Springer, 2007.

(89) Abb, M.; Wang, Y.; Papasimakis, N.; De Groot, C. H.; Muskens, O. L. Surface-enhanced infrared spectroscopy using metal oxide plasmonic antenna arrays. Nano Lett. 2014, 14, 346-352.

(90) Munk, B. A. Frequency Selective Surfaces: Theory and Design; Wiley: New York, 2000.

(91) David, M. Pozar; John Wiley \& Sons, Inc., 2005.

(92) Fan, S.; Joannopoulos, J. D. Analysis of guided resonances in photonic crystal slabs. Phys. Rev. B 2002, 65, 235112.

(93) Orfanidis, S. J. Electromagnetic Waves and Antennas. Media 2004, na.

(94) Ji, D.; Cheney, A.; Zhang, N.; Song, H.; Gao, J.; Zeng, X.; Hu, H.; Jiang, S.; Yu, Z.; Gan, Q. Efficient Mid-Infrared Light Confinement within Sub-5-nm Gaps for Extreme Field Enhancement. Advanced Optical Materials 2017, 5, 1700223. 